\title{
Cooperative handover algorithm based on auxiliary carrier in LTE-Advanced relay system
}

\author{
He Weiyang ${ }^{\text {a }}$,Zhao Jihong ${ }^{\text {a b }}$ \\ ${ }^{a}$ Xi'an University of Posts \& Telecommunications, China \\ ${ }^{\mathrm{b}}$ Nanjing University of Posts \& Telecommunications, China \\ he.weiyang@163.com, eeleeg@gmail.com
}

\begin{abstract}
Handover is one of the most important parts in mobility management. In the LTE-Advanced system, new technologies such as relay and carrier aggregation are introduced, which put forward higher requirements and challenges to the handover mechanism. By studying the handover process of the traditional LTE-Advanced system and LTE-Advanced Relay system, this paper proposes a cooperative handover algorithm with the consideration of the auxiliary carrier in LTE-Advanced relay system. This algorithm takes the advantages of wide spreading of low-frequency carrier, rich resources of high- frequency carrier, and in conjunction with relay technology and carrier aggregation technology. The propose algorithm reduces the handover outage probability and improve the throughput of the system.
\end{abstract}

Keywords- LTE-Advanced, Relay, Auxiliary Carrier, Cooperative Handover

\section{INTRODUCTION}

In the mobility management of mobile communication system, it will trigger the handover mechanism, when users lose the coverage of the current cell. Thus the user would handover from one cell to a neighboring cell. But with various sizes of different cell coverage, handover quality will be different. Therefore, outdoor wireless coverage-enhancing is one of the key performance indicators of the LTE-Advanced system. And the major researches on coverage-enhancing are through the adoption of relay technology to extend cell coverage area for the local cell shadow fading in affected areas and to cover corners signal to provide hotspots covered and indoor coverage in order to achieve the objective of enhancing the coverage ${ }^{[1]}$. So, the relay technology has become one of the key technology of next generation wireless communication system. In the process of the relevant technical standards of the LTE-Advanced system, carrier aggregation (CA), multiple user MIMO(Multi-input Multi-output), coordinated multi point transmission/reception (CoMP) and other new technologies are also introduced to obtain greater spectrum efficiency and throughput.

In the traditional LTE-Advanced system, the system uses hard handover technology, which is shown in figure 1 , while soft-handover mechanism is still under consideration ${ }^{[2][3]}$. Combined with the new technologies in the LTE-Advanced system, it also forms some of the other new handover mechanism. For example, ref. [2] proposed the joint handover mechanism of based on carrier aggregation. Ref. [4] made of semi-soft handover mechanism based on OFDM system and [5] proposed a part soft-handover mechanism, which not only reduces the interruption time, but also make the QoS of real-time business guaranteed. However, in the process of handover, the handover interrupt delay of these mechanisms was relatively large and the handover easily fail. In addition, when cell edge users hand over, the handover outage probability will be relatively large and the communication quality of the cell edge users will be influenced. After the introduction of relay stations to be LTE-Advanced system, the handover scenario is shown in figure 2 , and the handover process of the LTE-Advanced relay system based on the centralized and distributed had made a detailed statement in literature [6]. Compared with the traditional LTE-Advanced system, LTE-Advanced relay system ensures the edge community user performance and increases cell coverage. The relay station reduces the cost of operators to set up sites, increased system throughput, improves user data transfer rate. However, the LTE-Advanced relay system handover mechanisms only fit for single-carrier and small bandwidth 
systems. The future communications system requirements of multi-carrier and big bandwidth already have some limitations.

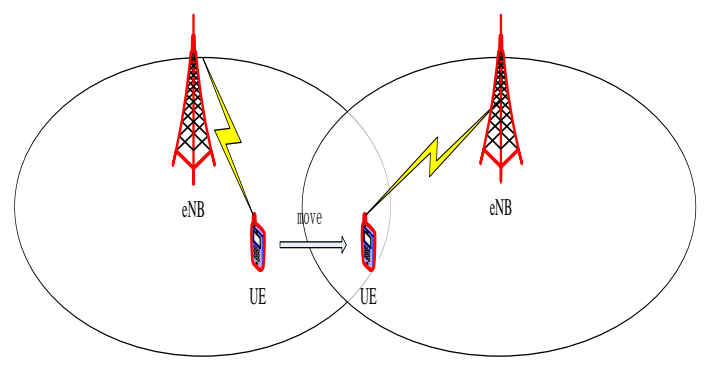

Figure 1. LTE-Advanced system handover scene graph

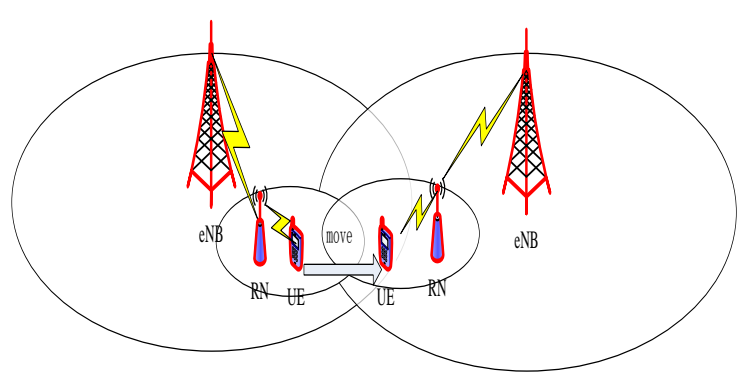

Figure 2. LTE-Advanced relay system handover scene graph

Since the LTE-Advanced system potential deployment spectrum is $450 \sim 470 \mathrm{MHz} 、 698 \sim 862 \mathrm{MHz} 、 790 \sim 862 \mathrm{MHz}$ 、

$2.3 \sim 2.4 \mathrm{GHz} 、 3.4 \sim 4.2 \mathrm{GHz} 、 4.4 \sim 4.99 \mathrm{GHz}$ and so on, it can be seen that the potential spectrum shows the tendency of high and low differentiation. High frequency band is obviously not as good as low frequency band in coverage, the ability to penetrate buildings and mobile ${ }^{[7]}$. At the same time, with the continued allocation of wireless resources, wider communication frequency will be achieved only in the high frequency band. In addition, the wireless signal in free-space attenuation proportional to the square of the frequency, higher frequency bands will bring a more serious space attenuation. Wireless cellular coverage radius will be reduced ${ }^{[8]}$. Based on the above analysis, combined with advantages of references [6] presented LTE system for relay-enhanced network handover algorithms, taking advantage of low-frequency carrier wide coverage and high-frequency carrier wave resource-rich, with carrier aggregation technology, we propose a cooperative handover algorithm based on auxiliary carrier (BOAC)in LTE-Advanced relay system. This algorithm enhances the area coverage, reduces the handover outage probability, and improves the system throughput.

\section{COOPERATICE HANDOVER ALGORITHM BASED ON AUXILIARY CARRIER IN LTE-ADVANCED RELAY SYSTEM}

This paper proposes collaborative handover algorithm based on auxiliary carrier, which uses the carrier aggregation technologies and the introduced auxiliary carrier activation/deactivation mechanism in the LTE-Advanced system. Among them, the carrier aggregation technology will aggregate the high and low-frequency carrier to meet system requirements of multicarrier and large bandwidth. At the same time, the use of auxiliary carrier activation/deactivation mechanism enables users to reduce handover interruption time, which improves users the quality of communications service.

\section{A. Algorithm Description}

In this algorithm, we take into account of the two-hop relaying system characteristics and high and low frequency coverage and carrier aggregation technology. The system is presented in figure 3 step 1 that consists of the three links: backhaul $\operatorname{link}(\mathrm{eNB}-\mathrm{RN})$, access $\operatorname{link}(\mathrm{RN}-\mathrm{UE})$ and direct link(eNB-UE).For far distance communication, we use low frequency carrier wave for transmission of access links information. As the distance is relatively close, backhaul and access links adopt the high-frequency carrier wave for transmitting information. Before the handover, as shown in the figure 3 step 1, base station simultaneous provides services to users by direct links and access links. UE receives information from these two links through different frequency band of non-continuous by carrier aggregation technology. When the user needs to handover, as shown in Figure 3, step 2, with the idea of "high-frequency handover, low-frequency auxiliary", high frequency carriers between relay stations and users are first handed over. In this process, low-frequency carriers between base stations and users continue to provide services. After the synchronization between the UE and the target RN, as shown in Figure 3, step 3 and 4, the target eNB and source eNB respectively carry out the low frequency carrier activation and deactivation mechanism, which makes the low-frequency carrier between source eNB and UE not provide service to UE. This handover strategy reduces the handover outage probability and handover interrupt delay, meanwhile improves user throughput. 


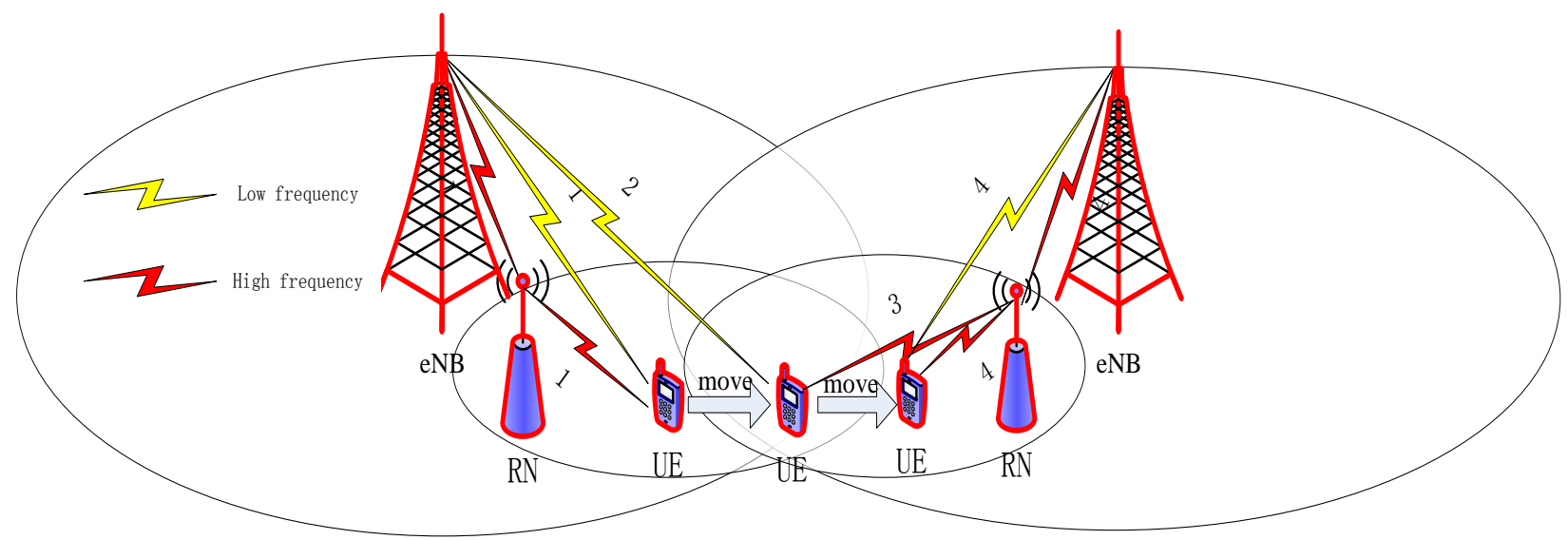

Figure 3. Cooperative handover strategy based on auxiliary carrier in LTE-Advanced relay system handover scene graph

\section{B. Algorithm Control Process}

According to LTE-Advanced relay system handover scene graph as shown in Figure 3, the handover algorithm are as follows:

1): The high frequency carrier wave channels between RN and UE and low frequency carrier channel between eNB and UE are measured by UE, and measurements reports are sent to the macro base stations.

2): eNB receives high-frequency and low-frequency carrier channel measurement report. Combined with the wireless resources management information eNB decides whether to handover: eNB will first determine whether the low frequency carrier signals meeting the conditions for continued data services for UE, if it does not, go to step 3; if the high-frequency carrier wave channels meet communication needs, do not handover, otherwise go to step 4.

3): When the high frequency carrier continued to provide data services to UE, if the low frequency carrier channel can't meet the condition for providing service, eNB will reselect low frequency carrier channel on all remaining candidate low frequency carrier channel, and this channel will replace the original low frequency carrier channel.

4): If there are not access the destination sites in neighboring eNB and all RNs which within neighboring eNB coverage area, then the high frequency carrier wave channels handover failed. Otherwise, select the best RN, and send handover instructions to target eNB that the best access target
RN corresponds. Target eNB allocates the corresponding low-frequency carrier channel for UE, and controls target relay station's assigned appropriate high-frequency carriers for the UE, Then target eNB sends handover request confirmation information that includes low frequency carrier channel and high frequency carrier channel to source eNB.

5): UE synchronized to the high frequency carrier wave channels which are allocated by the target RN. This time, low frequency carrier channel between source eNB and UE continuous providing data services for UE.

6): After synchronization, the target eNB and source eNB respectively execute the low-frequency carrier channel activation and deactivation mechanism. At the same time, target eNB will inform the gateway MME of the user's new location, so that the gateway can switch downlink path and will receive all packets send to target eNB.

7): Executing the rest of the process, release the resources of source eNB to UE and the source RN to UE, handover completed.

Due to the existence of two links arrive at the UE at the LTE-A relay systems, the control plane's signaling process is relatively complex. According to the algorithm described above, the signaling flow chart of cooperative handover algorithm based on auxiliary carrier in LTE-Advanced relay system control plane shown in figure 4 : 


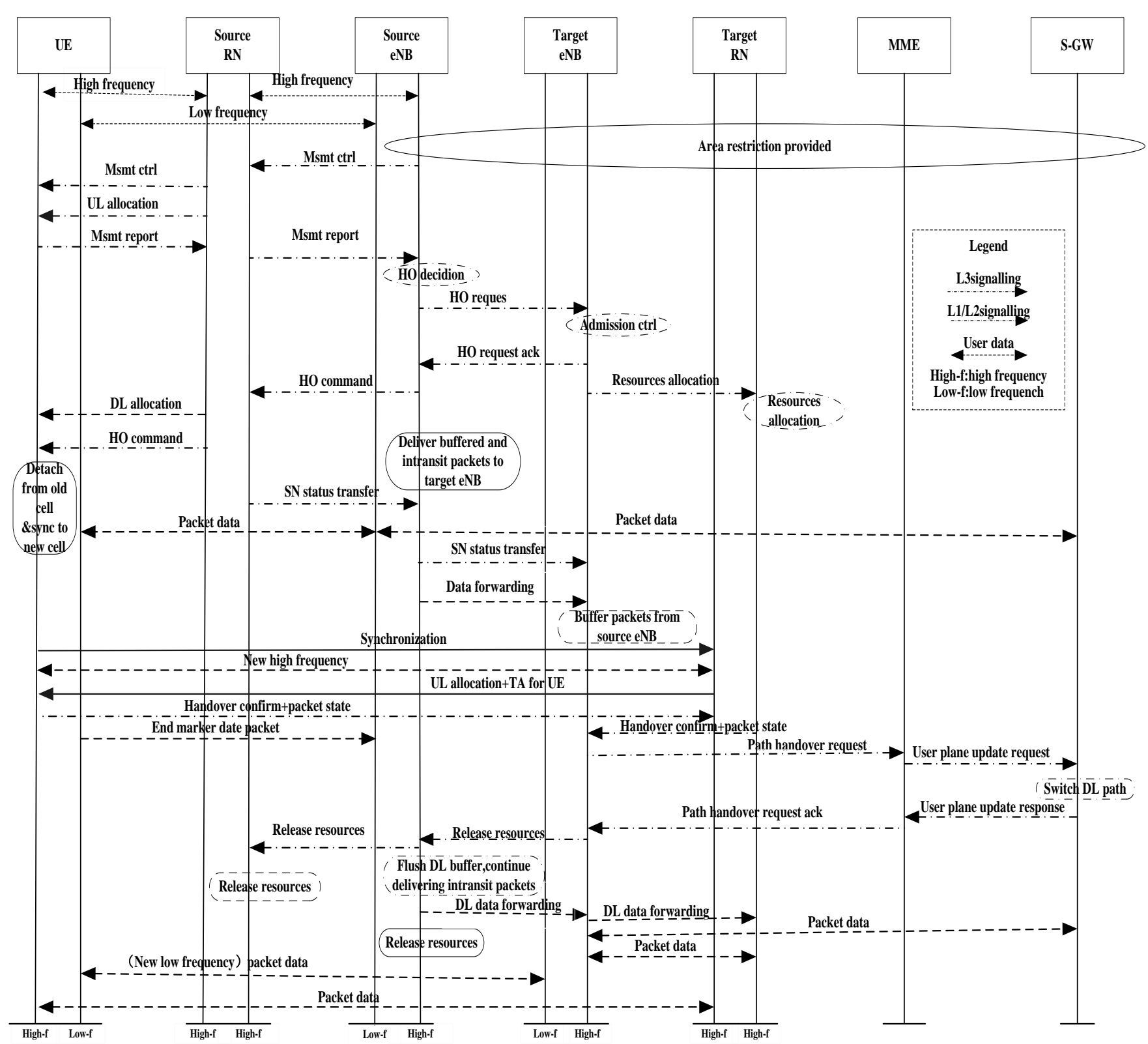

Figure 4. Cooperative handover algorithm based on auxiliary carrier in LTE-Advanced relay system control plane signaling chart

\section{PERFORMANCE EVALUATION AND ANALYSIS}

\section{A. User Throughput}

In this paper, the throughput of two-hops users(namely the edge users) will be evaluation(the throughput calculation formula main references [9]). Among them, the low-frequency carrier frequency is $800 \mathrm{MHz}$, high-frequency carrier frequency is $2000 \mathrm{MHz}$, and the bandwidths of both carriers are $10 \mathrm{MHz}$ respectively. After the use of carrier aggregation technology, the system bandwidth is expanded to $20 \mathrm{MHz}$.
Each cell is divided into three sectors, each sector using a directional antenna of 120 degrees, at the edge of each sector deployed two fixed relay stations and the fixed relay stations using Omni-directional antennas. Users from one cell move to another neighborhood cell along a straight line at a speed of 3 $\mathrm{km} / \mathrm{h}$. the path-loss of backhaul link keep constant. The users number of the cell are 10,15,20,25, 30 respectively.

TABLE I. SIMULATION PARAMETERS 


\begin{tabular}{|c|c|}
\hline parameters & Value \\
\hline Carrier Frequency & $800 / 2000 \mathrm{MHz}$ \\
\hline Bandwidth & $2 * 10 \mathrm{MHz}$ \\
\hline $\begin{array}{l}\text { Inter-Site Distance } \\
\text { (ISD) }\end{array}$ & $\begin{array}{l}\text { Case } 1: 500 \mathrm{~m}, \\
\text { Case } 3: 1732 \mathrm{~m}\end{array}$ \\
\hline eNB Transmit Power & $46 \mathrm{dBm}$ \\
\hline RN Transmit Power & $30 \mathrm{dBm}$ \\
\hline eNB Antenna Gain & $14 \mathrm{dBi}$ \\
\hline RN Antenna Gain & $5 \mathrm{dBi}$ \\
\hline Traffic Model & Full Buffer \\
\hline $\begin{array}{l}\text { Thermal Noise } \\
\text { Spectral Density }\end{array}$ & $-174 \mathrm{dBm} / \mathrm{Hz}$ \\
\hline $\begin{array}{l}\text { Minimum Distance } \\
\text { between eNB and } \\
\text { users }\end{array}$ & $35 \mathrm{~m}$ \\
\hline $\begin{array}{l}\text { Minimum Distance } \\
\text { between RN and users }\end{array}$ & $10 \mathrm{~m}$ \\
\hline $\begin{array}{l}\text { Moving Speed of the } \\
\text { user }\end{array}$ & $3 \mathrm{~km} / \mathrm{h}$ \\
\hline Number of cell users & $10,15,20,25,30$ \\
\hline Path Loss ${ }^{[10]}$ & $\begin{array}{l}\text { eNB-UE:PL }=119.7+37.6 \log _{10}(\mathrm{R}) \\
(800 \mathrm{MHz}) \\
\text { RN-UE:PL=128.1+37.6 } \log _{10}(\mathrm{R}) \\
(2000 \mathrm{MHz})\end{array}$ \\
\hline $\begin{array}{l}\text { Shadowing Standard } \\
\text { Deviation }\end{array}$ & $\begin{array}{l}\text { eNB-UE:8dB } \\
\text { RN-UE:10dB }\end{array}$ \\
\hline
\end{tabular}

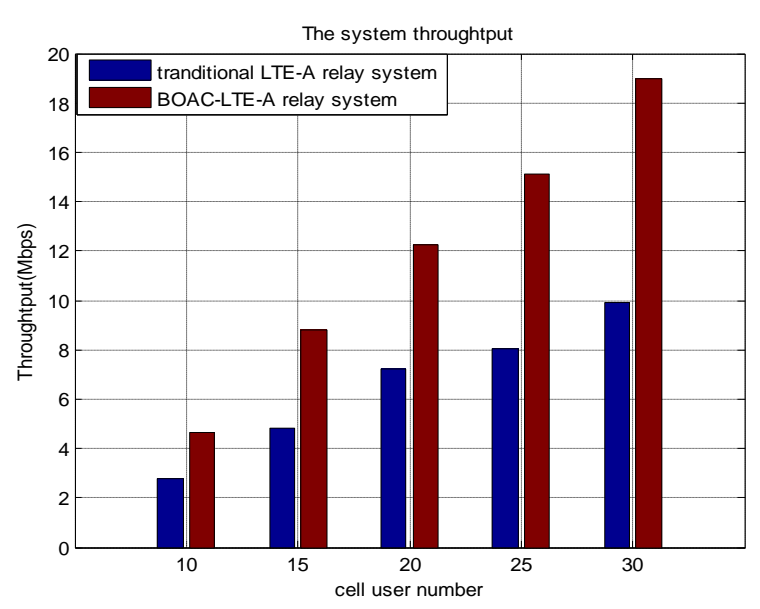

Figure 5. the system throughput

Fig.5 shows, compared to LTE-A relay system throughput, cooperative handover algorithm based on auxiliary carrier in
LTE-Advanced relay system significantly improved the throughput of the edge users. This is because the user constantly close to the edge of the cell, the path loss increases unceasingly at the edge of the cell, which makes the SINR decrease with the increasing of distance. Therefore, user throughput gradually decline. For the LTE-A relay system, due to the introduction of the relay station, the network reducing the distance between the user and the service eNB and makes the edge users received signal strength increase substantially, thus improving the UE data transmission rate. The user's throughput has been increased substantially. The proposed method not only introduces the relay technology, but also combines the carrier aggregation technology and the characteristics of low frequency carrier. In this way, the received signal strength of the cell edge user is increased significant, the path loss at the edge of the cell reduces, and further enhances the cell edge throughput.

\section{B. Handover outage probability}

This section compares the handover outage probability between the traditional LTE-A relay system handover algorithm and cooperative handover algorithm based on auxiliary carrier in LTE-Advanced relay system. In this simulation, standard deviation of shadow fading between the base station and the user is $8 \mathrm{~dB}$, and the standard deviation between the relay stations and the user is $10 \mathrm{~dB}$. Shadow fading between the base station and the relay station is $6 \mathrm{~dB}$. Assume that the system outage target threshold value $\varepsilon$ is $3 \mathrm{~dB}$, handover decision threshold value $\Gamma$ is 3 $\mathrm{dB}, \mathrm{BW} \mathrm{PRB}_{\mathrm{B}} / \mathrm{R}_{\mathrm{b}}=19200 \mathrm{bps} / \mathrm{Hz}, \mathrm{BW}_{\mathrm{PRB}}$ is the bandwidth of the physical resource block , $\mathrm{R}_{\mathrm{b}}$ is the bit rate, load rate of the cell is $25 \%$.

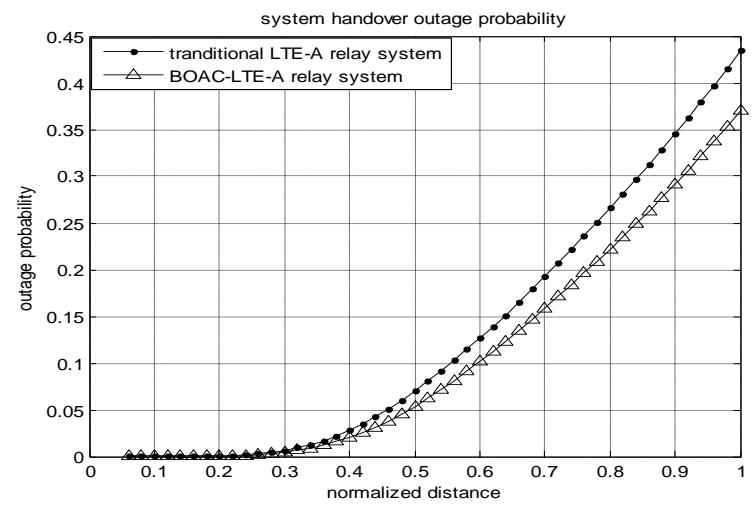

Figure 6. system handover outage probability 
Fig 6 shows, The traditional LTE-A relay cellular network handover outage probability is bigger, cooperative handover algorithm based on auxiliary carrier in LTE-Advanced relay system the handover outage probability is relatively smaller. This is because that the above two scheme are using the hard handover technology. That's to say, when the UE needs to handover, UE will first break with the connection with the source base station or relay station before establishing connection with the target base station or relay station. Relay stations decreases the distance between UE and connections sites, which effectively improves the link quality on the edge of the cell. However, for the cooperative handover algorithm based on auxiliary carrier in LTE-Advanced relay system, when the user hands over, after relay access link is disconnected, direct link between the base station and the user continues to provide service for the users. Thus it further reducing the handover outage probability.

\section{CONCLUDING REMARKS}

In this paper, we presented a cooperative handover algorithm based on auxiliary carrier in LTE-Advanced relay system. This algorithm mainly uses the characteristic of high frequency and low frequency whose channel propagations are different, We adopt the carrier aggregation techniques to aggregate high frequency and low frequency carriers in order to meet user demand for large bandwidth. At the same time, we introduce the cooperative handover idea of "high-frequency handover, low-frequency auxiliary" to reduce the handover interruption delay and the handover outage probability. The simulations show that users' handover outage probability decreases compared to the traditional LTE-Advanced relay system, and user' throughput improved.

\section{ACKNOWLEDGMENT}

This work is supported by the National Natural Science Foundation of China(No.61371087) and the National Science and Technology Major Projects Foundation "New Generation Broadband Wireless Mobile Communication Network"(No.2012ZX03001029-003

2012ZX03001008-003)

\section{REFERENCES}

[1] Yi Zheng Zhongnian Li Yafeng Wang. LTE - A system relay technology research; Modern telecommunications technology: 6th issue: 45-49
[2] Yang LEE,Jihong Zhao,Hua Qu.A Combined Handover Scheme for LTE-Advanced.IEEE,ICACT 2012:154-157.

[3] 3GPP TS 36.300,V11.1.0.Evolved Universal Terrestrial Radio Access (E-UTRA) and Evolved Universal Terrestrial Radio Access Network (E-UTRAN); Overall description; Stage 2 .2012.

[4] Hyungkeuk Lee, Hyukmin Son, Sanghoon Lee. Semisoft Handover Gain Analysis Over OFDM-Based Broadband Systems. IEEE Transactions on Vehicular Technology, 2009, 58(3):1 443 1 453.

[5] Junren Chang, Yajuan Li,Shulan Feng,et al. A Fractional Soft Handover Scheme for 3GPP LTE-Advanced System.IEEE.2009.

[6] Oumer Teyeb,Vinh Van Phan,Bernhard Raaf,Simone Redana. Handover Framework for Relay Enhanced LTE Networks.IEEE.2009.

[7] Jia Sheng Shiqiang Suo Hanyang Quan. 3GPP long term evolution (LTE) technology principle and system design. Beijing: People's posts and telecommunications publishing house, 2010.

[8] Biao Feng Yin Wang. LTE - the Advanced wireless relay network handover algorithm research,.Beijing university of posts and telecommunications, 2012,3.

[9] 3GPP TS 36.826 V0.13.0 Evolved Universal Terrestrial Radio Access (E-UTRA); Relay radio transmission and reception.2012.

[10] 3GPP TS 36.942 V10.3.0 Evolved Universal Terrestrial Radio Access (E-UTRA); Radio Frequency $(R F)$ system scenarios.2012.

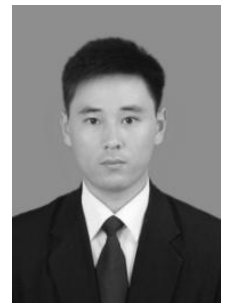

He Weiyang. was born in 1987.He is currently working toward the M.S. degrees in Xi'an University of Posts and Telecommunications. His research interests include wireless broadband network.

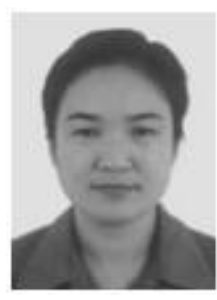

Zhao Jihong, was born in 1963, Ph.D., profe-ss or of Xi' an Jiaotong University and Xi'an University of Posts and Telecommunications. Her current research interests include wireless broadband network, mobile Internet, network management and control. 